\title{
Essais
}

ESSAIS

Revue interdisciplinaire d'Humanités

12 | 2017

Textes et contextes : entre autonomie et dépendance

\section{La publication multisite : un objet linguistique qui interroge les notions de texte et de contexte dans les environnements numériques}

Laetitia Émérit

\section{(2) OpenEdition}

Journals

Édition électronique

URL : http://journals.openedition.org/essais/3026

DOI : 10.4000 /essais.3026

ISSN : 2276-0970

Éditeur

École doctorale Montaigne Humanités

Édition imprimée

Date de publication : 15 juin 2017

Pagination : 173-190

ISBN : 979-10-97024-02-4

ISSN : $2417-4211$

Référence électronique

Laetitia Émérit, «La publication multisite : un objet linguistique qui interroge les notions de texte et de contexte dans les environnements numériques », Essais [En ligne], 12 | 2017, mis en ligne le 12 octobre 2020, consulté le 25 octobre 2020. URL : http://journals.openedition.org/essais/3026 ; DOI : https:// doi.org/10.4000/essais.3026 


\section{La publication multisite : un objet linguistique qui interroge les notions de texte et de contexte dans les environ- nements numériques}

\section{Laetitia Émérit}

Les publications multisites sont des objets communicationnels nativement numériques ${ }^{1}$ peu étudiés en linguistique que je souhaite aborder ici sous l'angle de la relation texte-contexte. Il s'agit de contenus (texte, image, vidéo, lien hypertextuels) qui sont diffusés (ou publiés) dans plusieurs environnements numériques de façon simultanée. Les publications multisites sont utilisées par des particuliers et des professionnels dans l'objectif de maximiser leurs communications avec les différents réseaux qu'ils entretiennent. On les retrouve sur tous les réseaux sociaux numériques (désormais RSN), mais également sur des sites d'annonces (vente, immobilier, emploi).

Dans cet article je me suis intéressée aux publications multisites présentes sur Facebook ${ }^{\oplus}$ et Twitter ${ }^{\bullet}$ qui font partie des RSN les plus utilisés en France en $2015^{2}$. J'ai choisi ces deux RSN car ils proposent tous les deux des outils intégrés de publication multisite renvoyant l'un vers l'autre ${ }^{3}$, (ce qui n'est pas le cas de Google + ) et qu'ils permettent de publier du texte (ce qui n'est pas le cas de YouTube).

Pour cela je m'appuierai sur des captures d'écran provenant de mes comptes Facebook $^{\oplus}$, Twitter ${ }^{\ominus}$ et Hootsuite ${ }^{\circledR}$. J'ai également réalisé un micro-corpus

1 Paveau M.-A., "En naviguant en écrivant. Réflexions sur les textualités numériques ", JeanMichel Adam, Faire texte. Frontières textuelles et opérations de textualisation, Presses universitaires de Franche-Comté, 2015.

2 Les RSN les plus importants en termes de nombre d'utilisateurs en France en 2015 sont : Facebook $^{\oplus}$ (30 millions), YouTube (23 millions), Google+ (10 millions) et Twitter ${ }^{\oplus}$ ( 6 millions) selon le blog du modérateur. http://www.blogdumoderateur.com/chiffres-2014-mobile-internet-medias-sociaux/.

3 Facebook $^{\oplus}$ et Twitter ${ }^{\circledR}$ proposent chacun une application permettant de reproduire simultanément les publications faites sur l'un ou sur l'autre. Il ne s'agit pas d'un outil commun, pour que toutes les publications apparaissent sur les deux sites l'utilisateur devra autoriser l'application de Facebook ${ }^{\oplus}$ à publier sur Twitter ${ }^{\circledR}$ et celle de Twitter à publier sur Facebook ${ }^{\oplus}$.

4 Les pseudonymes des utilisateurs de Facebook ${ }^{\circledR}$ et Instagram apparaissant dans les captures d'écran seront anonymisées en raison du fonctionnement semi-privé de ces sites. 
réunissant les publications de vœux de bonne année $2016^{5}$ postées sur ces deux $\mathrm{RSN}^{6}$ par les 10 personnalités politiques françaises les plus suivies sur internet ${ }^{7}$.

Intégrer au corpus ces deux ensembles de données, d'un côté les captures d'écran de mes comptes personnels et de l'autre le micro-corpus, me permettra de saisir la publication multisite dans son intégralité. J'ai souhaité focaliser le micro-corpus sur les vœux de bonne année 2016 postés par une sélection de personnalités politiques car il s'agit d'une occasion privilégiée de publications multisites. Les personnalités politiques (elles-mêmes ou leurs équipes de communication) pratiquent régulièrement la publication multisite. C'est-àdire qu'ils répètent généralement un contenu identique sur tous leurs réseaux afin de mieux diffuser l'information.

La thématique des souhaits de bonne année est une opportunité due au moment de constitution du micro-corpus. J'ai réuni les données en janvier 2016, les vœux étaient facilement accessibles sur les timelines ${ }^{8}$ des réseaux sociaux. Ils présentent également l'avantage d'être publiés par une grande partie des personnalités politiques et de posséder une forme attendue assez rigide "*bonne année 2016 » à partir de laquelle les variations sont facilement repérables.

L'objectif de cet article est de proposer une définition de la publication multisite telle qu'elle apparait dans le cadre des RSN, dans son rapport au(x) contexte $(s)^{9}$. Puis, par l'observation empirique de cet objet linguistique, d'analyser les enjeux de la relation texte-contexte dans le discours numérique natif.

Je commencerai par réinterroger la notion de contexte(s) en la mettant en perspective avec celle d'environnement numérique ${ }^{10}$ et en proposant une typologie des contextes liés aux publications sur les réseaux sociaux numériques.

Je poursuivrai en proposant une description de la publication multisite basée sur cette reconfiguration de la notion de contexte et sur des captures d'écran réalisées à partir de mes comptes personnels Facebook ${ }^{\oplus}$, Twitter ${ }^{\oplus}$ et Hootsuite ${ }^{\oplus}$.

5 Ce micro-corpus a été constitué le 06/01/2016.

6 Il s'agit de Facebook ${ }^{\circledR}$ et Twitter $^{\circledR}$ car Hootsuite ${ }^{\ominus}$ n'est pas un RSN, j'y reviendrai dans la suite de l'article.

7 Selon le classement du site Elus 2.0 consulté le 06/01/2016 à l'adresse suivante : http://www. elus20.fr/classement-politique-twitter-facebook/.

8 La Timeline est la page d'un réseau social sur laquelle on retrouve toutes les publications d'un membre par ordre antéchronologique.

9 Paveau M.-A., "En naviguant en écrivant. Réflexions sur les textualités numériques ", JeanMichel Adam, Faire texte. Frontières textuelles et opérations de textualisation, Presses universitaires de Franche-Comté, 2015.

10 Paveau M.-A., "Technodiscursivites natives sur Twitter. Une écologie du discours numérique ", Épistème (Revue internationale de sciences humaines et sociales appliquées, Séoul), 9, (2013a), p. 139-176. 
Puis, je terminerai sur l'analyse du rapport entre contexte, texte et publications numériques à partir des observations empiriques permises par le microcorpus de vœux 2016.

\section{Contextes et environnements numériques}

La pluralité représentationnelle du message, telle qu'elle se présente dans le cadre des publications multisites, demande de réinterroger la notion de contexte. De quoi parle-ton lorsque l'on parle du contexte d'une publication numérique?

Il existe deux niveaux de "contextes " associés aux publications numériques. Le premier, que j'appellerai contexte numérique pour faciliter l'explication, est lié au type de site, de technologie, aux choix de l'utilisateur, etc. Le second, disons le contexte communicationnel, est lié à la rédaction, à l'apparition et à la réception de la publication.

Chacun de ces deux niveaux de contexte est complexe et dépend de variantes différentes mais qui peuvent porter sur les mêmes objets. Par exemple, le " contexte numérique " d'une publication sur Facebook ${ }^{\oplus}$ peut être la page du journal d'un utilisateur sur lequel elle a été publiée. Dans ce contexte interviennent toutes les particularités du site mais également ses potentialités qui entrent dans le paradigme des structures numériques de type réseau social. Son " contexte communicationnel "d'apparition sera également cette page de journal du site mais il sera en lien avec les " contextes communicationnels » de rédaction et de réception qui ne seront pas nécessairement les mêmes en fonction du "contexte numérique " de la publication. Dans cet exemple Facebook ${ }^{\oplus}$ permet des contextes de rédaction (journal) d'apparition (journal + fil d'actualité) et de réception (journal et/ou fil d'actualité) différents.

Ces deux " contextes » sont enchâssés et interdépendants. S’il est possible de les démêler comme je viens de le faire pour décrire des publications simples, cela pose un réel problème lorsqu'il s'agit de publications multisites. Ce nouveau type de publication a pour particularité principale d'apparaitre dans plusieurs " contextes numériques ». Il devient donc nécessaire de les décrire plus finement ce que ne permet pas la polysémie du terme "contexte».

Pour résoudre cette situation, je propose d'associer la notion de contexte à celle d'environnement numérique, telle que la décrit Marie-Anne Paveau ${ }^{11}$ (2013), dans une perspective d'écologie discursive :

" J'appelle environnement l'ensemble des données humaines et non humaines au sein desquelles les discours sont élaborés. La notion d'environnement est pour moi une alternative critique à celle de contexte courante en analyse du discours, plutôt centrée sur les paramètres sociaux, historiques et politiques.

11 Paveau M.-A., "Environnement ", Technologies discursives [Carnet de recherche], (2013c), http://technodiscours.hypotheses.org/?p=311, consulté le 07/06/2015. 
Cette notion est cohérente avec une approche écologique de la production des énoncés, impliquant que l'objet d'analyse n'est plus seulement l'énoncé mais l'ensemble du système dans lequel il est produit. »

L'environnement numérique représente un ensemble, un lieu, composant l'écosystème à l'intérieur duquel les énoncés (ou les textes) apparaissent et évoluent. Cette notion d'environnement permet de mieux décrire les objets discursifs nativement numériques et de les placer dans une perspective écologique sans laquelle il ne peut y avoir de prise en compte de leur numéricité (au sens de leur composante technologique).

La notion d'environnement numérique recouvre donc celle de « contexte numérique » que j'ai utilisé plus haut. Elle se distingue de celle de cotexte ${ }^{12}$ que l'on pourrait envisager par l'implication de l'environnement sur le contenu. L'environnement numérique, contrairement au cotexte, a une action directe sur le texte (ou la publication), dans sa forme, dans sa présentation et parfois dans le contenu de l'énoncé.

Comme je l'ai évoqué dans l'exemple précédent, c'est l'environnement numérique qui détermine les potentialités, ou les modalités, qui seront constitutives d'une publication. Sur Twitter ${ }^{\circledR}$ par exemple les énoncés ne pourront pas dépasser 140 caractères, il s'agit bien d'une action directe de l'environnement sur le contenu.

On peut distinguer plusieurs niveaux environnementaux, je propose d'en différencier trois tels qu'ils apparaissent dans la modélisation ci-après :

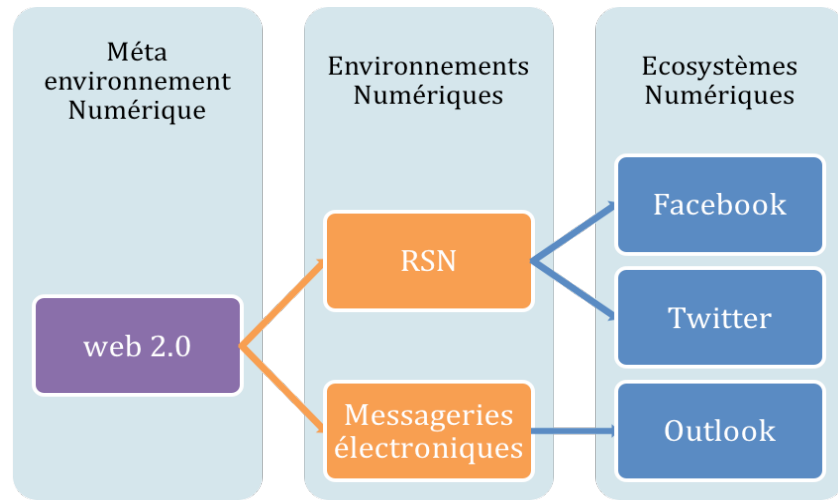

Fig. 1 : Environnements numériques

12 Kerbrat-Orecchioni C., "Le contexte revisité ", CORELA-RJC Cotexte, contexte, situation, hors-série $\mathrm{n}^{\circ} 11,2012$, http://corela.revues.org/2627. 
Le méta environnement numérique ${ }^{13}$ (web 2.0) ${ }^{14}$ est le niveau global (on pourrait le comparer à la terre pour filer la métaphore biologique). À l'intérieur de ce niveau il existe plusieurs types d'environnements (la mer, la montagne, la forêt, etc.) qui peuvent héberger chacun un certain nombre d'écosystèmes différents (un lac, un arbre, une grotte, etc.). Ces différents niveaux environnementaux sont reliés entre eux par l'utilisation qu'en ont les internautes qui est symbolisée dans ce schéma simplifié par les flèches. Chaque niveau environnemental est un lieu de passage qui permet d'accéder à un ou plusieurs autres environnements, les flèches permettent de représenter ce « mouvement».

Les réseaux sociaux numériques sont des environnements numériques au même titre que les forums de discussion, les services de messagerie électronique ou encore les serveurs d'écriture collaborative ${ }^{15}$. À l'intérieur de ces environnements numériques on peut distinguer plusieurs écosystèmes numériques, par exemple Facebook ${ }^{\oplus}$, Twitter ${ }^{\circledR}$, Instagram, etc., pour les RSN, Outlook et Gmail pour les messageries électroniques, etc.

La notion d'environnement n'est pas exclusive de celle de contexte. L'environnement (ou les environnements) est indépendant de la publication (textuelle ou multimodale). Mais la notion d'environnement ne permet pas de décrire finement des objets numériques comme, par exemple la publication multisite précisément parce que l'environnement existe indépendamment des publications. Or, pour décrire un objet comme la publication multisite qui est (j'y reviendrai) trans-environnementale, il devient important de trouver un outil théorique qui soit lié uniquement à la publication. La notion de contexte trouve alors une place, elle décrit les éléments entourant et interagissant avec la publication dans le paradigme de la publication et non celui de l'environnement.

Réserver la notion de contexte aux éléments directement liés aux publications en complément des aspects environnementaux permettra, par exemple, de décrire des phénomènes trans-environementaux ${ }^{16}$. Le contexte d'une publication est constitué de la configuration de son écosystème associée à un stade précis de son évolution numérique. Dans l'environnement numérique des RSN les trois stades de l'évolution d'une publication sont : celui de la rédac-

13 Il faudrait ajouter à ce premier niveau l'environnement numérique matériel qui se rapporte aux machines et écrans par lesquels les utilisateurs accèdent au numérique.

14 Herring, S. C., " Discourse in Web 2.0: Familiar, reconfigured, and emergent ", in D. Tannen \& A. M. Tester (Eds), 2013, Georgetown University Round Table on Languages and Linguistics 2011: Discourse 2.0: Language and new media (p. 1-25). Washington, DC: Georgetown University Press.

15 Zlitni S., Liénard F. (éds), La communication électronique dans la "société de l'information". Quels usages, quelles pratiques?, Le Havre, Klog Éd, 2012.

16 J'emploie ici « trans-environnemental » pour qualifier des objets dont la vision globale demande de prendre en compte plusieurs environnements numériques. 
tion (ou de la composition dans le cas d'une publication multimodale), celui de l'apparition et celui de la réception.

Je postule donc qu'il existe au moins trois contextes principaux (qui existent indépendamment de leur différenciation ${ }^{17}$ ) pour chaque publication : le contexte de composition (C1), le contexte d'apparition (C2) et le contexte de réception $(\mathrm{C} 3)$.

En fonction des potentialités (liées à l'environnement et/ou à l'écosystème dans lequel se situe chaque contexte) $\mathrm{C} 1, \mathrm{C} 2$ et $\mathrm{C} 3$ peuvent se superposer, se confondre ou se différencier. Il existe également des contextes secondaires en fonction des actions permises par l'écosystème sur les publications comme le contexte de modification ou le contexte de partage (entre autres). Ces contextes peuvent être considérés comme secondaires car ils ne sont pas systématiques, leur existence est dépendante des choix des utilisateurs et des potentialités offertes par l'écosystème.

Les trois captures d'écran ${ }^{18}$ ci-après représentent l'enchaînement suivant $\mathrm{C} 1=\mathrm{C} 2 \neq \mathrm{C} 3$ :

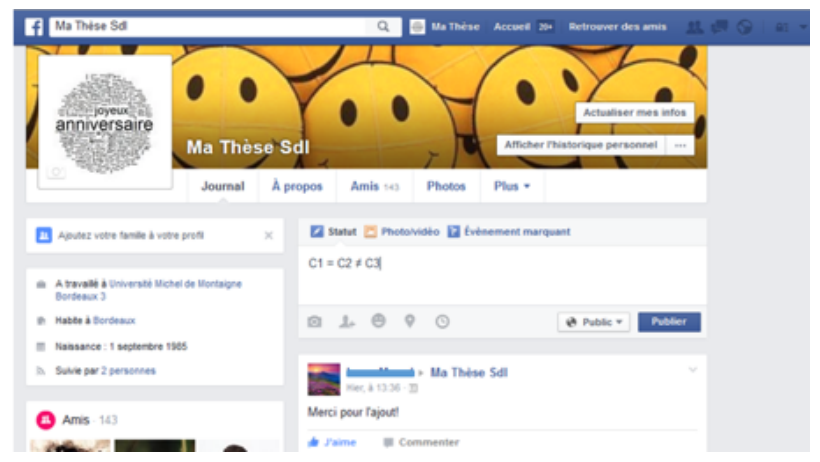

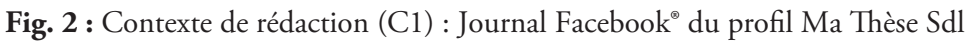

17 En fonction des affordances de l'écosystème et des choix des utilisateurs un ou plusieurs stades de la publication peuvent avoir des représentations identiques ce qui ne veut pas dire qu'ils n'existent pas de façon successive et différenciée.

18 Il s'agit de données construites en vue d'illustrer le fonctionnement que je souhaite décrire dans cet article, cette construction a été nécessaire car la collecte de données naturelles (ou semi-naturelles, demanderait de mettre en place un système de capture d'écran dynamique complexe permettant d'observer les pratiques de plusieurs locuteurs appartenant au même réseau. Si un tel corpus représente un réel intérêt scientifique il ne m’est pas possible de le mettre en ouvre dans le cadre de cet article. Sans ce corpus naturel il est impossible d'observer des données pour $\mathrm{C} 1$ et $\mathrm{C} 3$ qui ne soit pas capturées sur mes comptes personnels. Les trois captures d'écran ont été réalisées le 12 janvier 2016, les deux premières sur le journal du profil «Ma Thèse $S \mathrm{dl}$ " qui est un lieu de corpus ouvert et évolutif constitué dans le cadre de ma thèse de doctorat et la troisième sur le fil d'actualité de mon compte personnel sur Facebook ${ }^{\circledR}$. 


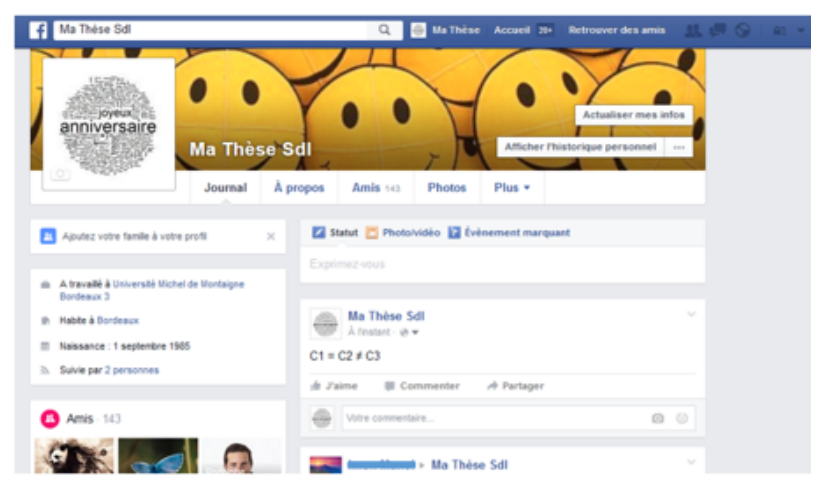

Fig. 3 : Contexte d'apparition (C2) : Journal Facebook ${ }^{\oplus}$ du profil Ma Thèse Sdl

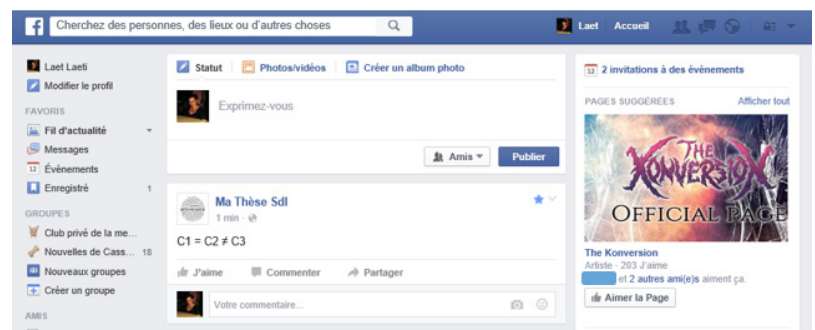

Fig. 4 : Contexte de réception (C3) : Fil d'actualité Facebook ${ }^{\bullet}$ du profil Laet Laeti

Dans cet exemple d'enchaînement le rédacteur compose son message directement sur son journal (Fig. 1), le message apparait sur le journal (Fig. 2) et le récepteur reçoit le message sur son fil d'actualité personnalisé par un algorithme en fonction de son activité sur le site (Fig. 3).

Ces trois contextes des publications dans les RSN se distinguent par une distribution complémentaire de trois traits : l'unicité, l'évolutivité et l'itération. Ces trois traits vont permettre (nous y reviendrons) de décrire la publication multisite. Le tableau 1 propose une synthèse de leur distribution pour C1, C2 et C3.

\begin{tabular}{|l|c|c|c|}
\cline { 2 - 4 } \multicolumn{1}{c|}{} & Unique & Évolutif & Itératif \\
\hline C1 & + & - & - \\
\hline C2 & - & + & - \\
\hline C3 & - & - & + \\
\hline
\end{tabular}

Tableau 1 : Traits distinctifs des contextes liés aux publications numériques

Le trait d'unicité ( + +/- Unique » dans le tableau 1) représente l'impossibilité, pour une seule publication, de posséder plusieurs contextes de même niveau. Le contexte de rédaction est le seul contexte principal à posséder ce trait.

Certains contextes secondaires peuvent posséder ce trait, c'est le cas par exemple du contexte de modification. Chaque modification d'une publication possède un contexte de rédaction unique puisqu'elle intervient dans un temps 
différent. C'est également le cas du contexte de partage. L'élément partagé, qu'il soit interne (partage d'une publication à l'intérieur d'un seul écosystème) ou externe (partage d'un élément extérieur à l'écosystème) est enrichi de données appartenant au " partageur " (pseudonyme, photo de profil) et du contexte techno-langagier (date et heure du partage, nombre de partages du même élément dans l'écosystème). Le contexte de partage est donc unique pour chaque partage, bien que l'élément partagé soit le même.

Il existe plusieurs contextes d'apparition possibles pour une publication dans les RSN : le profil, le fil d'actualité, le profil d'un autre utilisateur, les murs de pages ou de groupes ${ }^{19}$. L'apparition de la publication dans ces différents contextes, quel que soit leur nombre, se fait de façon synchrone, c'est-àdire qu'il s'agit de la même publication qui apparait dans des contextes différents, même à l'intérieur d'un seul écosystème.

Il existe autant de contexte de réception que de récepteur et de modalité de réception. Un utilisateur peut recevoir la même publication sur son profil et sur son fil d'actualité par exemple. De plus il est possible de recevoir une publication dans plusieurs méta-environnements. Un utilisateur peut par exemple recevoir une publication sur une application mobile et/ou sur le site internet.

Le trait d'évolutivité ("+/- Évolutif » dans le tableau 1) se rapporte à ce que devient le contexte une fois que le temps de l'action qui le lie à la publication est passé. $\mathrm{C} 1$ et $\mathrm{C} 3$ dépendent du rédacteur $(\mathrm{C} 1)$ et du récepteur $(\mathrm{C} 3)$. Le contexte de rédaction disparait pour laisser la place au contexte d'apparition et le contexte de réception est éphémère puisqu'il est lié à l'interaction entre le récepteur et la publication.

En revanche C2 est indépendant des utilisateurs lorsqu'une publication est publiée elle apparait sur le site, qu'un utilisateur soit là pour la recevoir ou pas. C2 peut évoluer dans le temps de deux façons. Si le rédacteur d'une publication modifie les paramètres de confidentialité le contexte d'apparition évolue puisque les modifications apportées aux publications sont rétroactives. C2 peut également évoluer automatiquement sous l'effet de l'environnement si celui-ci propose un archivage des données.

Les caractéristiques du contexte d'apparition varient en fonction des facteurs environnementaux et éco-systémiques dans lesquels il s'intègre. Dans la figure 5 on voit que le contexte d'apparition de la publication prise en exemple plus haut (Fig. 3) a évolué :

19 De façon automatique pour les deux premiers et en fonction des choix de publication du rédacteur pour les autres. 


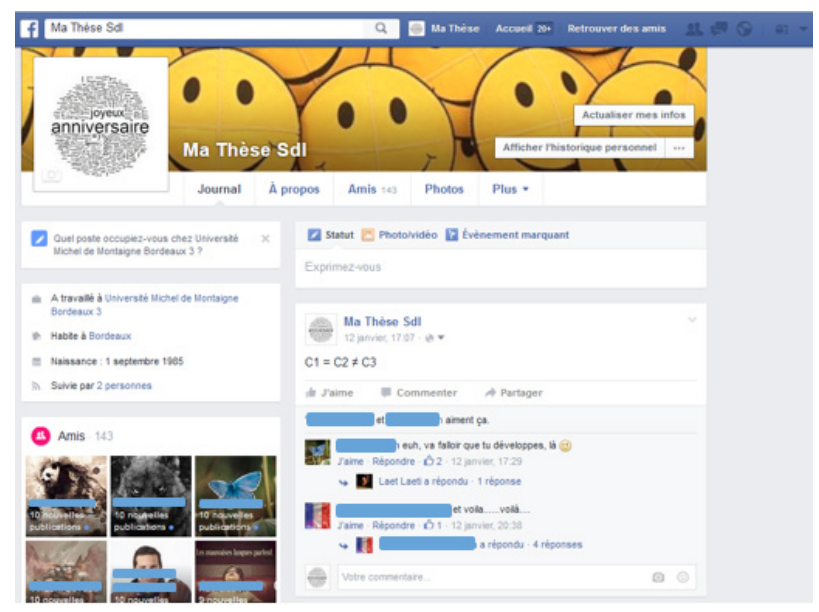

Fig. 5 : Contexte d'apparition sur Facebook ${ }^{\circledR}$ à $J+3$

Le trait d'itération («+/- Itératif » dans le tableau 1) se rapporte à la possibilité pour un contexte attaché à une publication à un moment $\mathrm{m}$ d'être renouvelé à un moment m'.

Le contexte de rédaction ne peut pas être renouvelé puisqu'il est éphémère, une fois la publication publiée il disparaît.

Le contexte d'apparition ne peut pas être renouvelé dans le cadre d'une publication faite dans un écosystème numérique unique ${ }^{20}$. Le trait itératif concerne chacun des contextes d'apparition d'une publication, de façon indépendante.

En revanche $\mathrm{C} 3$ réapparait à chaque fois qu'un utilisateur reçoit la même publication dans le même écosystème numérique, il possède donc le trait d'itération.

Cette reconfiguration de la notion de contexte, mieux adaptée à la réalité du terrain numérique, est nécessaire pour décrire les publications multisites. Ce qui les différencie des autres types de textualité nativement numériques étant justement ce rapport au(x) contexte(s) et aux environnements numériques, en particulier au contexte d'apparition.

\section{Contextes et publications multisites}

Dans le cas des publications multisites on retrouve les trois contextes principaux de la publication. Les contextes de rédaction $(\mathrm{C} 1)$ et de réception (C3) qui sont liés aux utilisateurs conservent les caractéristiques décrites ci-avant (Tableau 1). Par contre celles du contexte d'apparition (C2) varient en fonction des modalités qui permettent la publication multisite.

20 Je reviendrai sur cet aspect à propos de la publication multisite. 
Il existe en effet plusieurs méthodes pour réaliser des publications multisites et celles-ci ont des conséquences sur le contexte d'apparition.

Les publications multisites peuvent se faire à partir d'applications proposées par les RSN. Les utilisateurs agissent en amont de la publication en paramétrant leur compte sur un RSN pour le lier à leur compte sur un autre RSN. Cela signifie que la publication est rédigée dans un écosystème et publiée simultanément sur plusieurs RSN.

Dans ce cas tous les contextes de la publication sont intégrés au même environnement. On pourrait par exemple avoir la configuration contextuelle suivante $: \mathrm{C} 1=$ Facebook $^{\oplus} / \mathrm{C} 2=$ Facebook $^{\oplus}+$ Twitter $^{\circ}$.

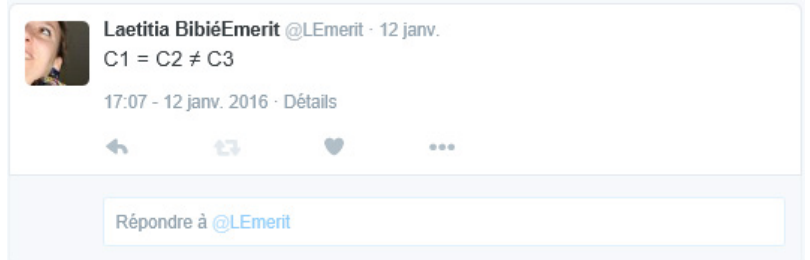

Fig. 6 : Contexte d'apparition Twitter ${ }^{\odot}$

La figure 6 montre le contexte d'apparition sur Twitter ${ }^{\circledR}$ de la publication représentée dans les figures 3 et 5 .

Les publications multisites peuvent également être gérées à partir d'un site ou d'un logiciel de centralisation comme Buffer ${ }^{\circledR}$ ou Hootsuite ${ }^{\oplus}$. Il s'agit généralement d'une stratégie de communication qui est mise en place par des entreprises, des bloggeurs ou des personnalités publiques (politiques entre autres) et qui vise à atteindre des publics différents de façon coordonnée et cohérente. L'utilisation de ces sites simplifie l'acte de publication puisqu'ils permettent de ne rédiger qu'une seule publication et de la distribuer sur tous les réseaux sélectionnés, de façon simultanée ou programmée. Le contexte de rédaction se situe donc en dehors des écosystèmes numériques d'apparition, il se trouve au niveau environnemental auquel appartient le site de centralisation.

Dans ce cas les étapes de la publication, et donc ses contextes, peuvent appartenir à des environnements différents, c'est ce que j'ai appelé plus haut la nature trans-environnementale de la publication. 


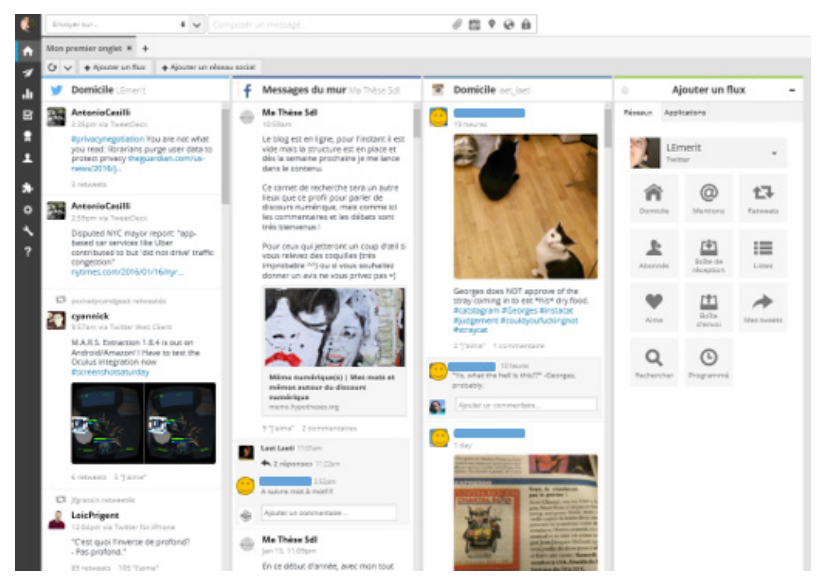

Fig. 7 : Page d'accueil Hootsuite

La figure 7 ci-avant montre la configuration de la page d'accueil de Hootsuite ${ }^{\oplus}$ sur laquelle l'utilisateur peut choisir les contenus qui apparaissent en les important de ses comptes sur les réseaux sociaux numériques ${ }^{21}$. Il est alors possible de rédiger des publications (encadré rouge dans la figure 7) et de sélectionner un ou plusieurs réseaux sociaux sur lesquels les faire apparaitre (encadré vert dans la figure 7).

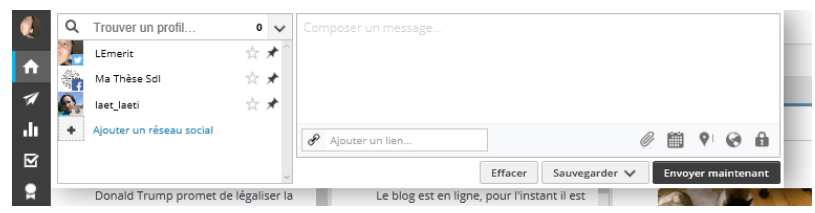

Fig. 8 : Contexte de rédaction (C1) sur Hootsuite ${ }^{\oplus}$

Hootsuite $^{\curvearrowleft}$ ne fait pas partie de l'environnement des réseaux sociaux numériques, pourtant comme on le voit dans les figures 7 et 8 il permet de publier des messages sur les RSN de façon simultanée mais également de façon programmée. Ces affordances ont une conséquence sur $\mathrm{C} 1$ qui fait partie de l'environnement numérique de Hootsuite ${ }^{\circledast}$ et surtout sur C2 qui possède le trait itératif lorsque l'utilisateur programme des publications différées. Cette évolution de C2 est représentée dans le tableau 2 par la ligne C2' :

21 Il y a une limite de 3 comptes dans la version gratuite mais il est possible d'en connecter une centaine dans la version payante. 


\begin{tabular}{|l|c|c|c|}
\cline { 2 - 4 } \multicolumn{1}{c|}{} & Unique & Évolutif & Itératif \\
\hline C1 & + & - & - \\
\hline C2 & - & + & - \\
\hline C2' & - & + & + \\
\hline C3 & - & - & + \\
\hline
\end{tabular}

Tableau 2 : Traits distinctifs des contextes liés aux publications numériques dans le cadre des publications multisites

C2' est itératif car la publication pourra être programmée pour apparaitre plusieurs fois dans le même environnement numérique même si c'est au sein d'écosystèmes différents.

Les outils théoriques que sont les notions d'environnement, d'écosystème et de contexte permettent de définir la publication multisite comme un élément nativement numérique différent de la publication simple. Mais quelles sont les conséquences de ces différences contextuelles sur les textes (ou les publications dans une perspective multimodale) ? Autrement dit en quoi la relation texte-contexte dans les publications multisites modifie-t-elle la forme du message ${ }^{22}$ ? C'est ce que je propose d'éclaircir au travers de l'exemple de l'analyse d'un micro-corpus de vœux de bonne année 2016 publiés sur Facebook ${ }^{\oplus}$ et Twitter ${ }^{\oplus}$ par les 10 personnalités politiques les plus actives sur les RSN en 2015 Selon le classement du site Élus 2.023.

Focaliser ce micro-corpus sur des personnalités politiques augmente les chances de rencontrer des publications multisites gérées à partir de sites comme Hootsuite ${ }^{\circledast}$. Ces plateformes sont généralement utilisées par les personnalités publiques, particulièrement celles disposant d'une équipe de communication, dans des visées stratégiques.

La parole politique doit aujourd'hui être présente dans les environnements numériques. La volonté d'assurer une présence médiatique importante des personnalités politiques les pousse vers la publication multisite. Celleci, particulièrement lorsqu'elle est faite à partir de logiciels de centralisation, permet de transmettre un message identique dans des environnements numériques différents en un minimum de temps.

22 Sandré M. (éd.), Analyses du discours et contextes, Limoges, Lambert-Lucas, 2009.

23 Consulté le 06/01/2016 à l'adresse suivante : http://www.elus20.fr/classement-politiquetwitter-facebook/. 


\section{La relation texte-contexte dans les publications numériques}

Dans la figure 9 ci-après j'ai réalisé une synthèse des informations concernant les publications du micro-corpus de vœux 2016 pour trois personnalités politiques. Je limite ici le tableau à Christiane Taubira, François Hollande et Christine Lagarde ${ }^{24}$ qui sont les seuls dont on peut présumer qu’ils ont eu recours à des outils (applications ou site de centralisation) de publication multisite ${ }^{25}$.

\begin{tabular}{|c|c|c|c|c|c|c|}
\cline { 2 - 7 } & \multicolumn{2}{c|}{ Christiane Taubira } & \multicolumn{2}{c|}{ François Hollande } & \multicolumn{2}{c|}{ Lagarde } \\
\cline { 2 - 7 } & Facebook & Twitter & Facebook & Twitter & Facebook & Twitter \\
\hline Partages & 398 & 306 & 1142 & 2451 & 150 & 178 \\
\hline Likes & 10078 & 487 & 28510 & 3045 & 2780 & 217 \\
\hline Date & $31 / 12 / 2015$ & $31 / 12 / 2015$ & $31 / 12 / 2015$ & $31 / 12 / 2015$ & $03 / 01 / 2016$ & $03 / 01 / 2016$ \\
\hline Heure & $20 h 53$ & $20 h 47$ & $20 h 12$ & $20 h 11$ & $06 h 03$ & $06 h 03$ \\
\hline Nature & texte & texte & texte & texte & texte & texte \\
\hline
\end{tabular}

Fig. 9 : Tableau récapitulatif microcorpus, vœux 2016

Ce tableau récapitulatif permet de mettre en évidence certaines des différences contextuelles entre les publications apparaissant sur différents écosystèmes, notamment le nombre de likes et de partages ${ }^{26}$ qui doivent être relativisés du fait du nombre d'utilisateurs bien plus élevé sur Facebook ${ }^{\oplus}$ (30 millions) que sur Twitter ${ }^{\oplus}(6 \text { millions })^{27}$.

Toutes les publications présentées dans la figure 9 sont de nature textuelle et apparaissent sur les deux RSN aux mêmes dates et à des horaires allant de très proches à identiques. Le décalage de quelques minutes entre les deux contextes d'apparition des publications de Christiane Taubira et de François Hollande montre qu'il ne s'agit pas de publications multisites, c'est-à-dire d'une seule publication apparaissant dans plusieurs écosystèmes numériques.

24 Dans cet article je ne poserai pas la question de l'identité réelle du rédacteur du message. Il est possible, dans le cas de personnalités politiques, que les messages soient élaborés par des services de communication. Il s'agit d'informations auxquelles je n'ai pas accès et qui ne rentrent pas en compte dans le contexte numérique de rédaction. Le rédacteur étant l'utilisateur possédant les identifiants du compte à partir duquel le message est rédigé. Par soucis de simplification, je considère les personnalités politiques qui signent les publications du microcorpus comme les rédacteurs présumés de ces publications.

25 Je reviendrai par la suite sur les publications d'autres personnalités politiques. Le micro-corpus compte également les publications de vœux de bonne année 2016 de Jean-Luc Mélenchon, Marion Maréchal Le Pen, Marine Le Pen, Rama Yade, Ségolène Royal, Nicolas Sarkozy et Najat Vallaud-Belcacem.

26 Ces boutons sont présents sur les deux sites, même si sur Twitter on parlera de " retweet " et non de partage il s'agit bien de fonctionnalités équivalentes. J'ai exclu les commentaires de ce tableau car Twitter ne donne pas accès directement au nombre total de commentaires pour un tweet et qu'un relevé manuel aurait été trop chronophage dans le cadre de la rédaction de cet article.

27 Toutefois cela ne change rien à la distinction contextuelle visible dans le tableau, raison pour laquelle j'ai choisi de ne pas faire de répartition proportionnelle. La popularité des personnages politiques français n'étant pas la question ici. 
Il serait également envisageable que ces publications aient été générées à partir d'un outil de publication multisite et programmées pour apparaitre avec quelques minutes de décalage dans chaque écosystème. Cette information est hors de ma portée mais elle serait révélatrice d'une stratégie de communication qui viserait à simuler des publications uniques par écosystème. L'analyse de stratégies communicatives visant l'utilisation des traces numériques pourrait être envisagée à partir de l'étude des publications multisites. Dans cet objectif il faudrait envisager un corpus plus important contenant plusieurs publications pour chaque personnalité et tenter de déterminer (présence d'un écart temporel régulier par exemple) s'il s'agit ou non d'une utilisation stratégique de ces outils ou s'ils ne sont pas en cause. Sans certitude à ce sujet je considèrerai dans cet article qu'il s'agit de publications différentes pour chaque écosystème.

Dans ces deux exemples il existe nécessairement plusieurs contextes de rédaction pour un contenu identique. Il faut donc admettre deux possibilités, soit une publication se définit par un contexte de rédaction unique et c'est l'action de publier qui fait la publication. Soit, à l'inverse, c'est le fait de publier un contenu unique qui caractérise la publication et il peut exister plusieurs contextes de rédaction.

La deuxième définition est problématique, principalement par ce qu'elle nie le caractère évolutif du discours numérique. Si on admet qu'une publication est définie par un contenu unique on doit également considérer que modifier une publication revient à créer une nouvelle publication ce qui semble assez improbable. Pour ces raisons je considère que Christiane Taubira et François Hollande ont publié des textes identiques sur des réseaux sociaux différents, il ne s'agit donc pas de publications multisites.

On peut par contre continuer à considérer que Christine Lagarde a réalisé une publication multisite, ce qui est confirmé sur la figure 10 puisque Hootsuite ${ }^{\oplus}$ précise les contextes de rédaction des publications dans ses flux. On peut lire que Christine Lagarde a rédigé sa publication sur Hootsuite ${ }^{\oplus}$ tandis que Christiane Taubira et François Hollande l'ont fait directement sur Twitter ${ }^{228}$.

28 Le site précise également la nature de l'environnement numérique global (Web, Android, etc.). Ces informations ne sont pas affichées sur Twitter ${ }^{\circledR}$ et ne sont pas toujours accessibles sur Facebook $^{\ominus}$. Hootsuite ${ }^{\ominus}$ (ou du moins la version gratuite que j'ai testée) ne propose pas ces informations pour les publications apparaissant sur Facebook ${ }^{\circledR}$. 


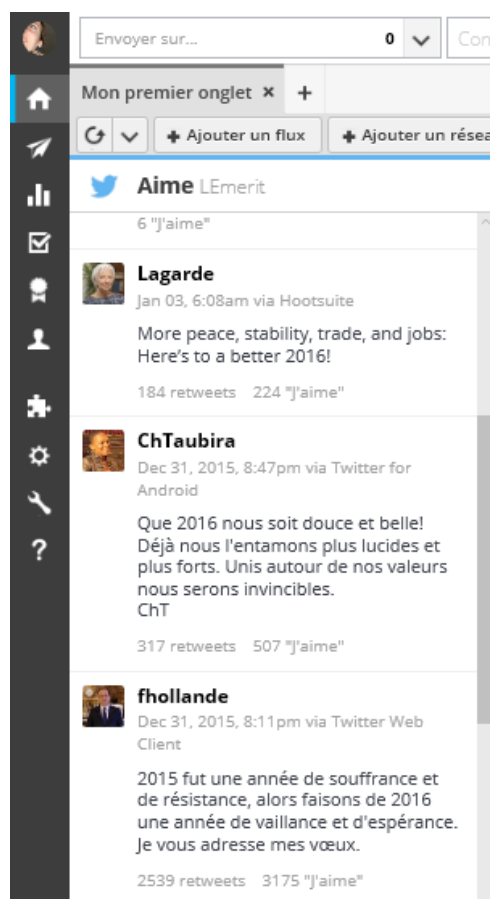

Fig. 10 : Tweets de vœux sur Hootsuite

Ces questions autour du rôle du texte et du contexte dans la définition des publications numériques obligent à redéfinir ces trois notions les unes par rapport aux autres.

Une publication est définie par son contenu qui peut être textuel et/ou multimodal. Un contenu textuel peut lui-même être plus ou moins métissé de technologie en fonction de son degré de numéricité intrinsèque ${ }^{29}$ mais aussi de l'écosystème numérique auquel il appartient. Dans le micro-corpus de vœux on peut citer par exemple les publications de Marion Maréchal Le Pen :

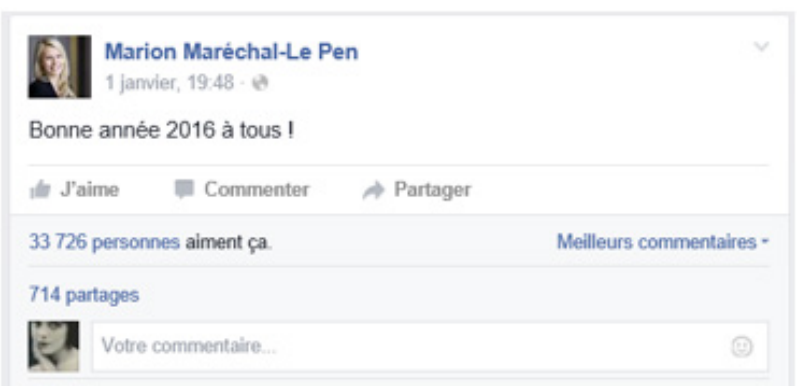

Fig. 11 : Vœux 2016, Facebook_Marion Maréchal Le Pen

29 Pour ces questions je renverrai à la typologie de Marie Anne Paveau (2015). 


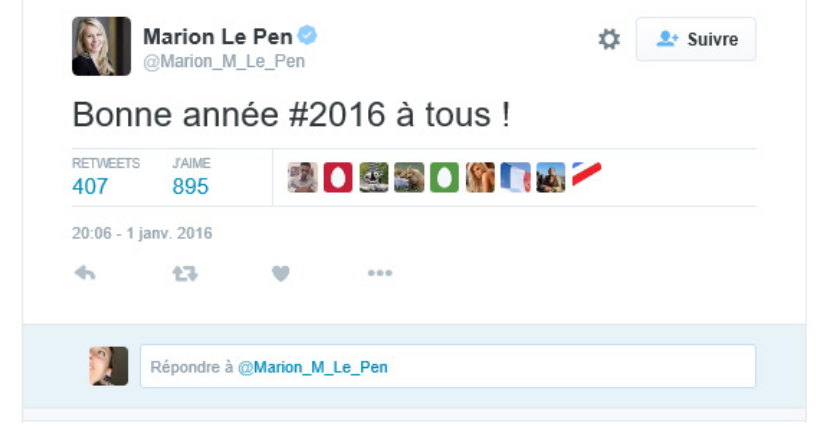

Fig. 12 : Vœux 2016, Twitter ${ }^{\circledR}$ Marion Maréchal Le Pen

Les publications reproduites dans les figures 11 et 12 se distinguent par leurs contextes d'apparition et par l'adjonction d'un signe dièse au texte publié sur Twitter. On se retrouve donc face à deux énoncés proches du point de vue du contenu linguistique mais dont le degré de numéricité est différent puisque l'introduction du signe dièse au début d'un groupe de signes typographiques (ici " 2016 ») déclenche une fonctionnalité du site qui en fait un hashtag ${ }^{30}$ et donc une forme cliquable.

L'ajout du hashtag n'ajoute pas directement de sens à l'énoncé mais il l'intègre dans son écosystème numérique d'apparition. On peut lui donner également une certaine valeur de reconnaissance sociale puisqu'il signale la maîtrise de l'outil, de l'environnement et des pratiques de la communauté. En ajoutant cet hashtag Marion Maréchal Le Pen signale son appartenance au groupe des utilisateurs de l'écosystème ce qui peut faire partie d'une stratégie de rapprochement avec les autres utilisateurs qui sont des électeurs potentiels.

Cet exemple montre comment le contexte de rédaction d'une publication peut influencer la forme et même la nature du texte. Il s'agit bien du contexte de rédaction ici puisque les deux RSN proposent cette fonctionnalité aux locuteurs, mais le hashtag est beaucoup plus pratiqué sur Twitter ${ }^{\circledR}$ sur lequel il est apparu en premier.

Aborder les publications numériques à partir de la notion de contexte permet de s'affranchir de la relation utilisateur-technologie numérique et de prendre en compte leur fonctionnement singulier. La description des contextes de rédaction, d'apparition et de réception des publications permet également de comprendre les différences entre publication et publication multisite.

30 C'est-à-dire un mot balise permettant de centraliser toutes les publications contenant le même mot balise. 
Pour cela il faut accepter d'appeler contexte(s) les éléments liés aux différentes étapes de la vie de la publication ce qui s'éloigne d'une forme de contextualisation qui serait sociolinguistique ou technolinguistique (Paveau, 2013) que je place plutôt du côté de l'environnement et du cadrage ${ }^{31}$. Tout en se positionnant dans une perspective écologique de l'étude du discours numérique il faut donc réserver la notion de contexte aux éléments dont l'existence dépend de la publication et non de son rapport à l'environnement dans lequel elle se situe. C'est à cette condition que la notion de contexte(s) permet de décrire les éléments trans-environnementaux comme les publications multisites. La description de ces éléments nativement numériques permet, comme je l'ai montré dans cet article au travers des exemples de publications issues du micro-corpus de vœux 2016, de replacer la relation texte-contexte dans une situation d'interdépendance.

Cet article ne se veut pas exhaustif, il s'agit plutôt d'une réflexion en élaboration sur un élément constitutif du discours numérique, la publication, qui reste encore méconnu et qui est susceptible d'évoluer. Il serait intéressant d'interroger la relation texte-contexte-publication sur un corpus plus large, tant par le nombre des publications que par leur diversité. Cela permettrait peutêtre, par exemple, de construire une typologie plus complète des contextes secondaires de la publication numérique que je n'ai fait qu'ébaucher ici.

\author{
Laetitia Émérit \\ UMR 5263 CLLE-ERSS \\ Université Toulouse Jean-Jaurès \\ emerit.laetitia@gmail.com
}

\begin{abstract}
Résumé
La publication numérique est une forme techno-langagière (Paveau, 2013) qui demande de repenser les notions de texte et de contexte tels qu'elles apparaissent dans les environnements numériques. La publication multisite est une forme récente et complexe de publication numérique qu'il est impossible de décrire sans proposer un cadre théorique et un métadiscours adaptés. Cet article, en proposant une définition de la publication multisite et une typologie des contextes de publication, amorcera une discussion sur ces notions. L'observation empirique, à partir de données collectées sur les réseaux sociaux numériques Facebook ${ }^{\circledast}$ et Twitter ${ }^{\odot}$, permettra d'analyser la relation texte/contexte dans le discours numérique natif.
\end{abstract}

\title{
Mots-clés
}

Environnement numérique, discours numérique, contexte, publication multisite, analyse du discours numérique, interactions par écran, réseaux sociaux numériques.

31 Goffman E., Les cadres de l'expérience, Paris, Les éditions de minuit, 1991. 


\section{Abstract}

Online publication is a techno-discursive form (Paveau, 2013) that requires rethinking the notions of text and context as they appear in digital environments. Multisite publication is a recent and complex form of online publication that is impossible to describe without proposing a theoretical framework and a new meta-discourse. This article, by proposing a definition of multisite publication and a typology of publication contexts, aims to discuss those two notions. An analysis of textcontext relationship in native digital speech is carried out on the basis of empirical observation, from data collected on social networks Facebook ${ }^{\circledR}$ and Twitter ${ }^{\circledR}$.

\section{Keywords}

Digital environment, digital speech, context, multisite publication, digital speech analysis, screen interactions, social networks. 